$\xi=-1$

\title{
Analysis of Driver Drowsiness Detection using EEG and EOG
}

\author{
Omprakash K Firke ${ }^{1 *}$, Dr. Manish Jain ${ }^{2}$ \\ ${ }^{1}$ PhD Scholar, SRK University, Bhopal \\ ${ }^{2} \mathrm{HOD}, \mathrm{EC}, \mathrm{RKDF}$ IST, Bhopal \\ *Email: omprakashphirke@gmail.com
}

\begin{abstract}
This paper propose here a resolutely oriented approach to the study of the driver in order to detect the driver drowsiness starting from physiological information (related to the brain activity) and video (related to the ocular activity). The goal of this work is to develop a system for automatic detection of driver drowsiness in the driver from electroencephalographic (EEG) (describing brain activity) and video of the driver. This approach is motivated by the fact that driver drowsiness physicians mainly work from brain and visual data to detect driver drowsiness. In addition, the complementarity of brain and ocular activities seems to indicate that the contribution of cerebral information would improve the reliability of the camera-based approaches (thus using only the visual cues) used for the automatic detection of the decline of vigilance.
\end{abstract}

Keywords: EEG; EOG; ECG; Driver Drowsiness; OSS Criteria.

\section{Introduction}

It's been a few years now that drowsy driving is recognized as a factor significant accident. If it is difficult to estimate the exact proportion of accidents attributable solely to fatigue, various international research estimates that it would be involved in $20 \%$ of accidents.

Recently, some drowsiness detection devices have appeared. Their goals: to help drivers to assess their tiredness and convince them to stop on time. While some devices integrated in the vehicle seem efficient, they remain reserved for people who can allow the purchase of a new vehicle equipped with such systems. Many "portable" systems, of tenmore affordable, are therefore proposed as an alternative to integrated systems.

\subsection{Driver Drowsiness}

Before all things, it seems important to give as precise a definition as possible of the phenomenon which interests us.

Intermediate state between wakefulness and sleep during which the body has its faculties of observation and analysis very reduced [1].

Indeed, this is the transition between the two fundamental states of awakening and sleep. This transition is unintended and accompanied by a decrease in alertness, that is, the ability to focus on a task. However, care must be taken to differentiate driver drowsiness from fatigue. The term fatigue is a depletion of the body after sustained activity too long. It exists in two forms: physical and mental. These two forms are not necessarily linked. Fatigue requires rest, which is not necessarily synonymous with sleep. It does, however, decrease the ability to maintain focus and focus because the body needs rest, so there are fewer resources availa- ble. Driver drowsiness is therefore only a consequence of fatigue. The consequences of driver drowsiness at the physiological level are numerous. A study by the United States Departmentof Transportation for Federal HighWay Administration (FHWA) identifies these different consequences [2]:

- Lowering of attention,

- Increased time for information processing and decisionmaking,

- Increased reaction time to critical events,

- More variable and less effective control responses,

- Lower motivation and performance,

- Decrease in psychophysiological activity,

- Increased feelings of drowsiness and fatigue,

- $\quad$ Reduced vivacity.

In view of these different consequences, it is clear why driving in this state represents a danger for the driver and other users, as well as the challenge of developing a system capable of preventing driver drowsiness. Mechanisms such as circadian and homeostatic cycles (physiological cycles governing wakefulness and sleep), sleep inertia (time required for each individual to recover all their cognitive abilities upon awakening), micro-sleep (Sleep episode of a few seconds) as well as the driver's environment (time of day, weather conditions, driving time, etc.) can promote driver drowsiness, which only complicates this phenomenon.

For many years, research has been conducted to better understand this risky state and to implement systems for its automatic detection. Two types of approaches have been proposed to study this phenomenon. The first approach, the oldest, focuses on the behavior of the vehicle. Its purpose is to detect the abnormal behavior of 
the vehicle on the road caused by driver drowsiness. For this, various indices such as the position of the vehicle on the road, the movements of the steering wheel or the pressure force on the different pedals have been studied in order to prevent risky behavior related to driver drowsiness. This approach, however, remains very dependent on the road, road traffic and driving habits of the driver.

The second approach focuses on driver behavior. In this case, the goal is to detect the physiological and behavioral signs of driver drowsiness in the driver. Studies on the physiological signs at the level of brain activity and eyepieces were conducted to better understand and characterize this problem of driver drowsiness. It is also from these physiological signals that doctors work to quantify the level of vigilance in a patient. More recently, the use of onboard cameras in the vehicle has made it possible to develop detection systems based on visual cues of the decline in alertness such as blinking, yawning or the direction of the driver's gaze. However, no system has proved sufficiently effective at the moment to be commercialized and the physiological approach remains the most effective today to characterize driver drowsiness.

During these different works, several problems were raised, the main one being the difficulty of proposing a precise definition of driver drowsiness and its characteristics. In addition, driver drowsiness is manifested differently in different individuals, which increases the difficulty of defining a common frame of reference for the detection of this phenomenon. This latter finding, coupled with the fact that no approach has proved sufficiently relevant, has led to attempts to merge different techniques to improve driver drowsiness detection systems. Several biomedical signal processing techniqes are presented in [49]-[63].

\section{Problem Domain}

Indeed, driver drowsiness results from many complex factors that interact with each other. Moreover, if these factors favor the decrease of vigilance, everyone does not react in the same way and there are many inter-individual differences at this level [3]. The difficulty in defining driver drowsiness is related to the fact that it is a transition between two states and not a state in itself. Indeed, we have presented here the various factors as well as the effects of driver drowsiness but it is difficult to establish the precise characteristics. In fact there is no common alphabet of driver drowsiness that allows definitively defining whether a person is a victim of driver drowsiness or not, as the rules of Rechtschaffen\& Kales [4] to define the different phases sleeping for example. The work of Dinges [5], however, showed that physiological indicators, in particular brain and eye activities, are the best indicators of this state of transition between wakefulness and sleep. Indeed, Renner \&Mehring [6] have suggested that the study of brain activity can identify the failure in the treatment of information and that the study of ocular activity can identify the insufficiency perception skills. We will begin by focusing on electroencephalographic activity to build our detection system.

Driver drowsiness Scales have been proposed by specialized doctors to assess the level of awakening of a patient but none has yet been standardized. These include the approach developed by the Karolinska Institute called Karolinska Drowsiness Scale or KDS [7] or that developed by Muzet et al. [8] called Objective Sleepiness Scale.

The study shows that the "portable" devices tested did not allow drivers a better awareness of his state of fatigue and the associated risks:

- Often confused because intimately linked it is important to differentiate fatigue from drowsiness.
- Fatigue is a physical or intellectual impairment that in the case of fatigue flying usually translates as difficulty staying focused. Fatigue requires rest.

- Sleepiness is an intermediate state between awake and sleep, which in practice translated by the difficulty to stay awake. Drowsiness requires sleep.

\subsection{Electro-Encephalography (EEG)}

Sensors on the head record the activity of the brain that breaks down into several waves. These are the alpha and theta rhythms that are analyzed to determine the state of fatigue of the subject. The degree of fatigue can be determined by the alpha / theta balance [9]. Interference may be influenced by movements of the body, head, or eyes [10].

Electroencephalography (EEG) is the measurement of the electrical activity of the brain by electrodes placed on the scalp, often represented in the form of a trace called electroencephalogram [11].

Typically, EEG is described in terms of rhythmic and transient activities. The rhythmic activity of the EEG is divided into frequency bands. These different frequency bands are above all a matter of nomenclature and have been defined according to the biological significance of the different rhythms. It is possible, however, that some bands change a few Hertz from one author to another. EEG activity is mainly studied in the [1-30] $\mathrm{Hz}$ band because below and above this band, the EEG tends to be very noisy (when recorded under standard clinical conditions).

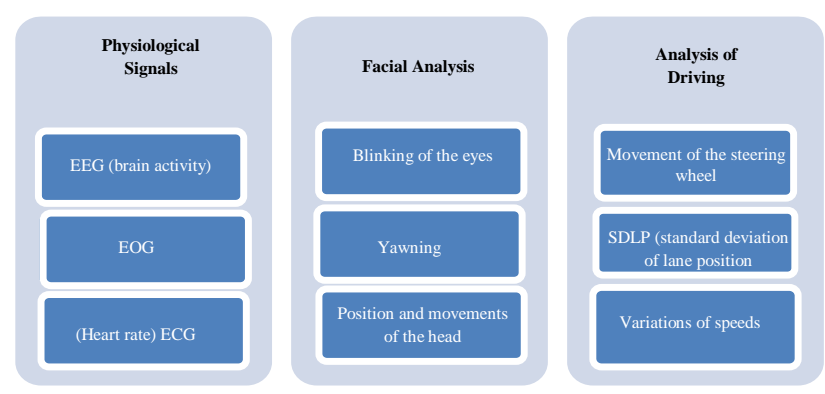

Fig. 1: The main scientific indicators of fatigue and distracted driving

Here is a brief presentation of the different frequency bands present in the GET:

Delta The activity $\delta$ corresponds to the frequency band [0.5-4] Hz. This activity is usually composed of high amplitude waves that appear in the frontal region in adults and parietal in children. This activity is mainly present in adults during deep sleep and in babies.

Theta: The activity $\theta$ corresponds to the frequency band [4-8] Hz. It is most common in children, drowsiness in adolescents and adults, and inactivity.

Alpha: The activity $\alpha$ corresponds to the frequency band [8-12] Hz. It occurs mainly in the parietal regions of the head but mainly on the dominant side and also in the central areas when resting. This activity is characteristic of relaxation.

Beta: The $\beta$ activity corresponds to the frequency band [12-30] $\mathrm{Hz}$. It is present on both sides of the brain symmetrically. It consists of low amplitude waves and appears more clearly in the frontal area. This activity is characteristic of awakening, active reflection and concentration.

Gamma: The $\gamma$ activity corresponds to the frequency band above $30 \mathrm{~Hz}$. It appears during certain cognitive tasks or motor functions and is difficult to record under standard clinical conditions. Figure 2 shows a second EEG signal then all the components of this signal, corresponding to the different frequency bands. 
Traditionally, the EEG is registered according to the 10-20 system presented in Figure 2. This system takes its name from the fact that the adjacent electrodes are placed at $10 \%$ or $20 \%$ of the total distance between the front and back and between the right and left of the skull, as shown in the figure. The value of the voltages measured ranges from approximately $10 \mu \mathrm{V}$ to $100 \mu \mathrm{V}$.Sampling often occurs around $250 \mathrm{~Hz}$.

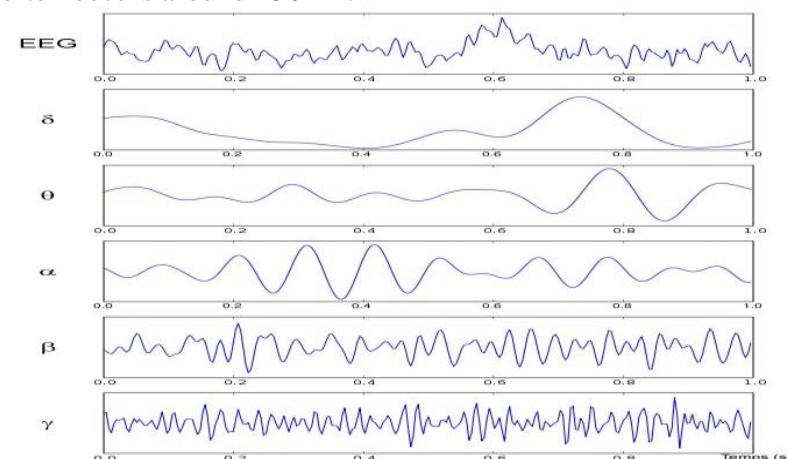

Fig. 2: EEG signal broken down according to its different frequency bands

\section{Manifestations of driver drowsiness in the EEG}

In view of the physiological significance of the different EEG frequency bands, it appears that the bands likely to be characteristic of driver drowsiness are the $\theta$ bands whose activity corresponds to drowsiness and inactivity, as well as the $\alpha$ band which corresponds to the rest and the closing of the eyes. Indeed, several studies $[12,13,14]$ have highlighted that the decrease in vigilance is characterized by an increase in electrical activity in the $\alpha$ and $\theta$ bands. Recent studies by. $[14,15,16]$ revealed that the $\alpha$ band is particularly characteristic of the decline of vigilance.

Moreover, we note that the $\beta$ band is characteristic of awakening and concentration. The decline in vigilance is also characterized by a decline in activity in the $\beta$ band. This decline in activity has been demonstrated by several studies [17]. However, the decrease in $\beta$ activity in case of driver drowsiness must be qualified following the work of [18]. Indeed, their study has shown that the decrease in alertness can be accompanied by an increase in $\beta$ activity due to an increase in concentration to try to compensate for the appearance of driver drowsiness.

Several scales have been proposed to evaluate the level of awakening but none has been standardized. Two scales of evaluation particularly caught our attention. The first is the method developed by the Karolinska Institute called Karolinska Drowsiness Scale or KDS [19]. This method proposes to quantify the wakefulness level from the EEG and EOG readings. The method of notation is inspired by the rules of notation of the phases of sleep developed by Rechtschaffen\& Kales [20]. The data are analyzed by segment of 20 s themselves divided into sub-segments of $2 \mathrm{~s}$. Whenever a sign of driver drowsiness is found on one of the sub-segments the score assigned to the segment is incremented by 10 . Thus the maximum score is 100 and the subject will be considered a victim of driver drowsiness if the score of the segment is higher at 50 . The second scale that interested us is the one developed by Muzet et al. [21). This is an Objective Sleepiness Scale (OSS). This OSS is separated into five levels of alertness ranging from 0 (fully awake) to 4 (very advanced driver drowsiness). Decisions are made every $20 \mathrm{~s}$ depending on the length of the puffs of activity $\alpha$ and $\theta$ present in the EEG as well as the speed of eye movements and blinks. These criteria are presented in Table 1.
Table 1: OSS Criteria

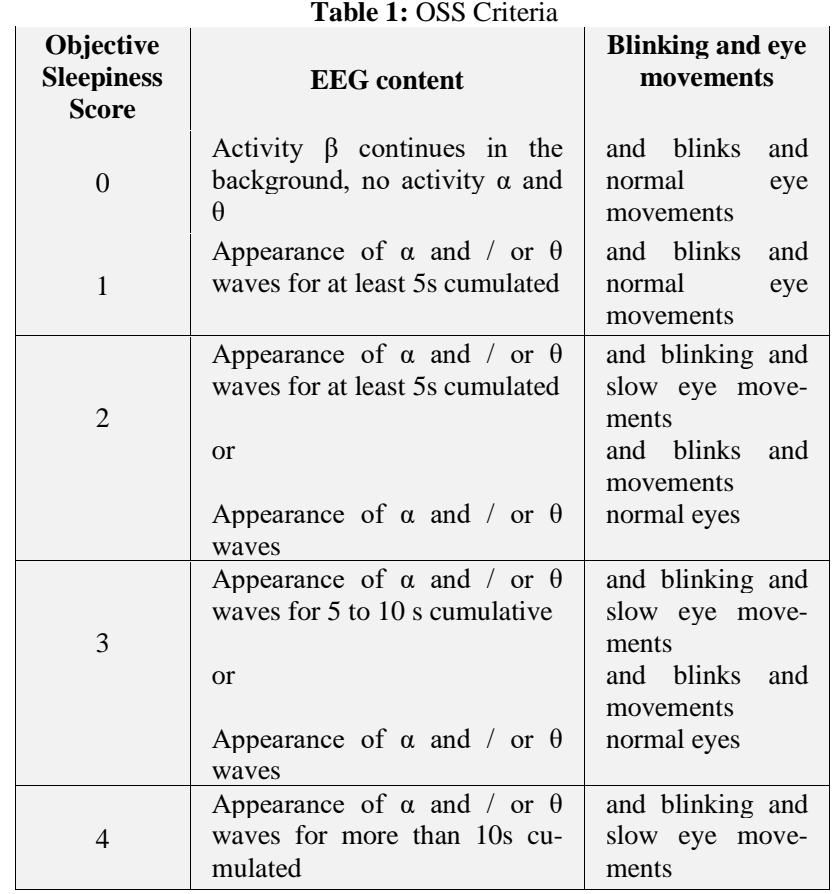

The two scales presented are used by expert physicians to visually assess the level of alertness from a person's different EEG and eye data. First, both scales confirm Renner \&Mehring [22] about using both brain activity and blinking to quantify the level of alertness.

This tends to prove that the problem of the characterization of driver drowsiness remains a delicate subject and that there is no universal scale.

That said, the use of EEG by expert physicians to quantify the level of alertness of subjects encourages us to propose an algorithm that is based on the EEG to detect moments of driver drowsiness. The purpose of this algorithm will be detection, in the as far as possible, the different EEG criteria characteristic of driver drowsiness, in particular the hause of $\alpha$ activity.

\subsection{Detection systems}

The majority of EEG driver drowsiness detection systems rely on the analysis of the frequency content of EEG signals. Indeed, as we have seen in the previous section, driver drowsiness mainly results in an increase in activity in the $\alpha$ and $\theta$ bands. The detection systems therefore seek to analyze the frequency content in order to detect these increases.

There are two methods for analyzing the frequency content of a variable frequency signal: the Short Time Fourier Transform (STFT) and the Wavelet Transform (WT). The principle of the STFT is to compute the Fourier transform, i.e. to compute the frequency content of the signal, on a sliding window, i.e. a part of the signal, the goal being to have a time-frequency representation. The WT for its part is a time-frequency analysis performed from a function called a basic wavelet that can be contracted or expanded to specify the characteristics of the signal that it is desired to detect. This technique was developed by Mallat [23]. A comparison between the uses of these two techniques in the context of the EEG study was carried out by Kiymik et al. [24]. It emerges from this work that STFT is more suitable for real-time applications and that WT is more suitable for applications requiring high resolution. In practice, most of the work was done using STFT. This technique has not yet been tested on actual driver drowsiness signals.

There are two types of method for analyzing the frequency content of the EEG: techniques with learning and no learning. Examples of supervised learning-based techniques include the use of neural networks. The neural network is a learning algorithm composed of 
a connected neural structure associating inputs to one or more outputs. When trained with driver drowsiness data, the neural network will allow the learning of a "black box" type model able to detect the decline of vigilance. The main difference between all approaches using neural networks lies in the nature of the inputs used as well as the type of network used. Authors of [25] suggested applying a PCA to a three-channel EEG spectrum to drive a multilayer neural network on the main component spectrum.Authors of[26] estimated the EEG spectrum by both STFT and WT to drive their network. Their technique has not been tested on real signals.Authors of[27] used a single parieto-occipital EEG channel (P4-O2) to educate two different networks. A network of SOM (Self-Organizing Map) type is used for the detection of driver drowsiness while another network of type LVQ (Learning Vector Quantization) is used for the detection of artifacts. [28] proposed to apply a network of neurons on the bands $\alpha, \theta, \beta$ and $\delta$ of an EEG spectrum obtained by WT in order to detect the following states: "awake", tired "and "sleeping".

Other classification techniques have also been used, such as Hidden Markov Model (HMM). The HMM [29] is a statistical model in which the modeled system is assumed to be a Markovian process of unknown parameters. A Markovian process is a process that estimates the future state from the only information of the present state and not past ones. This is still a technique requiring learning. The HMM is applied to an eight-dimensional vector where each dimension represents a particular band of the EEG spectrum.

These different techniques give good results, especially neural networks, but require learning on a large number of data in order to develop the model of driver drowsiness detection. In practice, this is not always feasible.

Other techniques that do not require learning also exist. We can cite the regressive models. The goal is to approach the signal with a mathematical model that is updated using past signal values. Hypov states

\section{Comparison with Literature}

Lin et al. [30] proposed to monitor driving performance, that is, the driver's ability to maintain the vehicle in the middle of the road, using a linear regression model on a two-channel EEG. When the model is driven and tested on the same session, they get a correlation of $r=0.88$ between their model and driving performance. However, this result falls to $r=0.7$ when the model is trained and tested on different sessions. Thus, we see that this method needs to be parameterized for each driver since the model estimated on one driver does not necessarily work very well on another. Lin et al. managed to improve these results by using an ICA on a 33-channel EEG [30] before applying their linear regression model. They then get a correlation of $r=0.88$ between their estimate and the actual driving performance on the test session. Nevertheless, two reservations can be made to this method. It requires a very large amount of data and channels to be trained and has been tested on only five topics.[31] obtain $92 \%$ good driver drowsiness detections by driving a neural network on the EEG spectrum recorded on the $\mathrm{P} 4-\mathrm{O} 2$ channel, however this good result is obtained on the model's learning data and falls back at $76 \%$ good detections on validation data, and again the method was only tested on a limited number of drivers [32] get an impressive 97\% of good detections with However, Rosipal et al.[33] reach $77 \%$ good driver drowsiness detections by using a GMM on the spectral content of their ELISA regression model. a converted EEG in the form of coefficients of an autoregressive model. This study was conducted on a large number of drivers but also requires a long period of training. Without drive and driver independent, $\mathrm{Pal}$ et al. [Pal08] obtained a correlation of $r=0.78$ between their indices obtained by the Mahalanobis distance and the driving perfor- mance of the driver. A summary of these results is presented in Table 2.

Table 2: Summary of the comparison of our results with those of the literature concerning the detection of driver drowsiness from the cerebral signs

\begin{tabular}{|l|l|l|}
\hline Authors & Results & Technique \\
\hline$[30]$ & $82.5 \%$ & MCT and fuzzy fusion on $\alpha$ and $\beta$ \\
\hline$[31]$ & $76 \%$ & Neural networks \\
\hline$[32]$ & $\mathrm{r}=0.88$ & Linear regression \\
\hline$[33]$ & $76 \%$ & Gaussian Mixture Model \\
\hline$[34]$ & $\mathrm{r}=0.88$ & Distance from Mahalanobis to $\alpha$ and $\theta$ \\
\hline
\end{tabular}

The advantage of our method, in addition to a good rate of detection of driver drowsiness states, is that it does not need to be adapted or trained. The threshold used for detection remains the same for each driver. Our method has the great advantage of operating from a single EEG channel. The artifact detector is used to determine the quality of the channel to avoid false detections due to disturbances. Moreover, the results are reliable because the method has been tested on a large number of drivers.

\section{Manifestations of Driver Drowsiness in the EOG}

Electrooculography (EOG) is the measure of the electrical activity of the eye. Thus, the EOG can be used to record eye movements and blinks. The eye can be considered as an electric dipole, the positive pole being the cornea and the negative pole the retina. The EOG is in fact the measure of the difference in electrical potential between these two poles, which can vary between 0.4 and $1 \mathrm{mV}$. When the eyes are at rest, the EOG measures a constant voltage, called baseline. The sign of change depends on the direction of movement. In addition, the amplitude of the measurement is proportional to the amplitude of the movement of the eye [35].

The EOG is measured by placing electrodes around the eyes. It is necessary to be able to differentiate the horizontal and vertical movements of the eye because the blinks are only vertical movements of the eyelids. The electrodes are placed vertically on either side of the eye for the vertical EOG (EOG-V). For the recording of the horizontal EOG (EOG-H), the electrodes are placed on the outer sides of the eyes. We also place an electrode in the middle of the front which will serve as a reference [36].

\subsection{Driver Drowsiness Detection Systems by Blinking Analysis}

It is clear that the ocular information retrieved by the EOG is a good indicator of driver drowsiness. Indeed, it can be recalled that the EOG is used by medical experts to evaluate the visual signs of driver drowsiness. The EOG has therefore naturally been subject to automatic driver drowsiness detection systems. Authors of [37] proposed a vigilance level detection system based on blink duration and frequency parameters as well as amplitude-velocity ratio. The average level of these variables is learned at the beginning of the recording, the system detects blinks that are too far away from these levels. Note that blinks less than $0.5 \mathrm{~s}$ are not taken into account. Learning techniques have also been used to classify different levels of vigilance from the EOG such as data partitioning analysis, the use of which has been suggested [38] or support vector machines. Authors of [39] Proposed using a model of Gaussian mixtures on Lissajous curves obtained from the horizontal and vertical channels of the EOG as well as movements of the head.

Recording an EOG on a driver is not necessarily obvious, especially since the electrodes must be placed very close to the eyes which can hinder the driver. Blink detection approaches using video have therefore been developed to detect eye movements. The use of video, however, introduces problems of image processing such as the location of the face or eyes. To overcome this problem, some studies have used infra-red (IR) lighting that pro- 
duces a very easily detectable reflection in the eyes. There are also driver drowsiness detection techniques based solely on IR oculography [40].

As we have seen previously, there are several techniques for extracting information from blinks using EOG or video. We are interested here in the link that exists between the various quantities estimated and the signs of driver drowsiness, that is to say how to use these quantities in the context of the detection of driver drowsiness.

One of the visual signs of relatively simple driver drowsiness to use is the detection of too long eye closure times. This is to alert the driver when the driver closes his eyes too long. Several studies have proposed detecting closures by segmenting the iris of the eye on the video [41, 42 and 43]. Indeed, the size of the iris decreases until disappearing when the eye is closed. Other studies have proposed to detect blinking by following the evolution of the upper eyelid and the lower eyelid, the blinks corresponding to the decrease in the gap between the two eyelids. Finally, methods have suggested detecting blinks by correlation between the bounding box of the eye and a closed eye model [44].Thus the correlation is maximum when the eye is completely closed. These different techniques all have in common to alert the driver only in case of prolonged closure of the eyes, that is to say when the eye is considered closed for more than five images in a row.

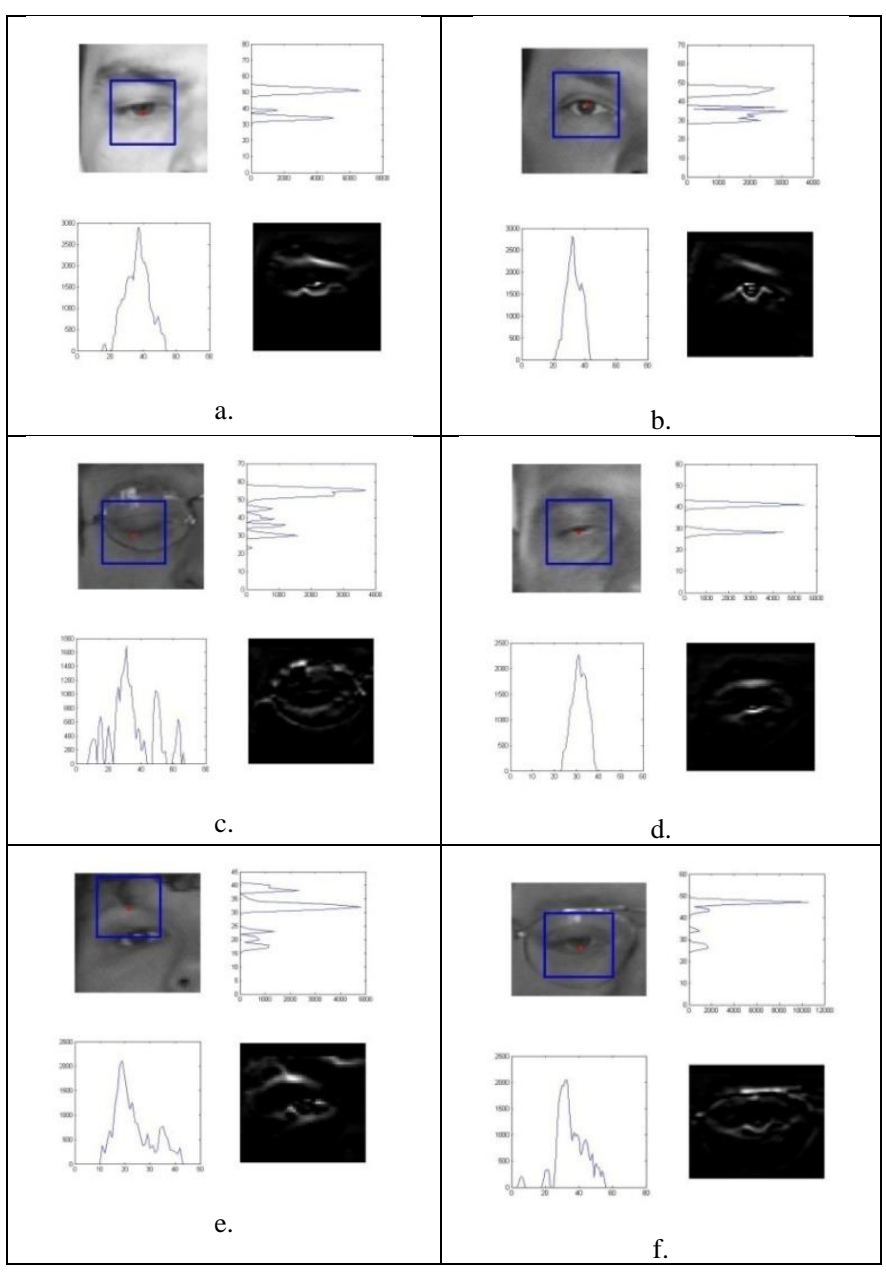

Fig. 3: Review of existing techniques

In addition, these systems alert the driver when the driver keeps his eyes closed too long, that is to say when the driver is already in a state of driver drowsiness advanced (even asleep at the wheel), which seems a little late. Indeed, a driver drowsiness detection system is more effective if it warns the driver before he falls asleep at the wheel, although it is important to wake him if it happens.
The second type of technique consists of more complete multivariable approaches. A study by Johns [45] proposed using the amplitude-velocity ratio to detect driver failure moments up to $60 \mathrm{~s}$ in advance. This ratio is calculated from an IR camera detection. It has been proposed to standardize velocity. Indeed, the velocity decreases with the decrease of vigilance whereas the amplitude changes very little. The advantage of using this report is that it does not need to be calibrated.

\subsection{Electrodermal Activity (EDA)}

The use of electrodermal activity as an indicator of the driver's driver drowsiness has long been the subject of studies. In 1966, the national road safety organization in France already asserted that the correlation in electrodermal activity and the level of vigilance had been validated scientifically. Since then, other studies have relied on the use of EDA as an indicator of vigilance and more specifically on the most relevant electrodermal indicators [46].

\subsection{Facial Analysis}

PERCLOS: The percentage of eyelid closure (PERCLOS) consists in measuring the percentage of time during which the eyes are closed at least $80 \%$ on the pupil during a certain period of time. An individual is considered fatigued when the PERCLOS exceeds $80 \%$ ([47]). This indicator seems reliable enough to determine the state of fatigue of an individual but the systems measuring the PERCLOS face a difficulty however: the brightness could make the detection difficult but also directly influence the blinking [48].

\section{Conclusion}

The detection of the eyes is carried out by a method using both gradient and projections. This method allows the detection of the eyes whatever their state. Blink detection is then performed from static and dynamic energy signals calculated on the area of the eye.

The comparison between blink characterization by EOG and video analysis shows that a fast camera approach can effectively replace traditional EOG analysis. The fast camera approach ( $\geq 100 \mathrm{fps})$ is also justified by the significant improvement in the results obtained in its use compared to those obtained with a standard 30fps camera. It has been shown that several parameters related to the detection of driver drowsiness such as duration, duration at $50 \%$, frequency and closing speed of the eye can be extracted from the video analysis with the same precision as on the EOG. On the other hand, certain parameters extracted from the video do not seem correlated with their homologues extracted from the EOG. However, depending on the application we make, they could still be used to detect the decline in vigilance. This is the case of the amplitude-velocity ratio which is strongly correlated whereas neither the amplitude nor the velocity show any significant correlation. Similarly, although performance is slightly improved when video sampling is increased, the improvement obtained is not significant enough to determine the choice of sampling. Once again, it is the application that will determine the choice of this parameter. However, it has been shown in this study that the use of a fast camera has a distinct advantage over the use of conventional 30fps sampling. In our case, the use of a sampling rate of 200fps seems the best choice to achieve a driver drowsiness detection system. It has been found in this paper that the problem of driver drowsiness is not a simple problem. Indeed, because driver drowsiness is a transition between the two states of awakening and sleep, it is difficult to define specific characteristics. Despite this, physiological features of driver drowsiness have been identified, giving rise to scoring rules of different levels of alertness, although these rules are not standardized and remain relatively subjective.

Based on the comparison of averages applied to the relative powers of the $\alpha$ and $\beta$ bands of the EEG. A high amplitude artefact 
detection system is also presented. It is combined with the driver drowsiness detector to detect in real time the periods of time when the EEG signal is unreliable (because polluted by artifacts). The artifact detector uses a single threshold that is independent of the driver.

EEG in order to detect signs of failure in the ability to process information and by studying blinks to detect signs of decline. Perceptual abilities. It now seems sensible to study how driver drowsiness can be characterized at the level of blinking. The fusion of the EEG and EOG approaches should make it possible to obtain a complete driver drowsiness detection system.

\section{Acknowledgement}

The authors would first like to thank the PhysioNet, for providing EEG images. Also, they would like to thank the reviewers and editors for their very helpful suggestions and insightful comments which have resulted in an improved paper.

\section{References}

[1] Lee, M. and Kim, J., Kwungpook National University IndustryAcademic Cooperation Foundation, 2017. Vehicle control system for providing warning message and method thereof. U.S. Patent 9,566,909.

[2] Oh, J., Washington, S.P. and Nam, D., 2006. Accident prediction model for railway-highway interfaces. Accident Analysis \& Prevention, 38(2), pp.346-356.

[3] Sahayadhas, A., Sundaraj, K. and Murugappan, M., 2012. Detecting driver drowsiness based on sensors: a review. Sensors, 12(12), pp.16937-16953.

[4] Anderer, P., Gruber, G., Parapatics, S., Woertz, M., Miazhynskaia, T., Klösch, G., Saletu, B., Zeitlhofer, J., Barbanoj, M.J., Danker-Hopfe, H. and Himanen, S.L., 2005. An E-health solution for automatic sleep classification according to Rechtschaffen and Kales: validation study of the Somnolyzer $24 \times 7$ utilizing the Siesta database. Neuropsychobiology, 51(3), pp.115-133.

[5] Van Dongen, H., Maislin, G., Mullington, J.M. and Dinges, D.F., 2003. The cumulative cost of additional wakefulness: doseresponse effects on neurobehavioral functions and sleep physiology from chronic sleep restriction and total sleep deprivation. Sleep, 26(2), pp.117-126.

[6] Renner, G. and Mehring, S., 1997. Lane departure and drowsiness-two major accident causes-one safety system. In MOBILITY FOR EVERYONE. 4TH WORLD CONGRESS ON INTELLIGENT TRANSPORT SYSTEMS, 21-24 OCTOBER 1997, BERLIN.(PAPER NO. 2264)

[7] Hu, S. and Zheng, G., 2009. Driver drowsiness detection with eyelid related parameters by Support Vector Machine. Expert Systems with Applications, 36(4), pp.7651-7658.

[8] Rogé, J., Pébayle, T., Lambilliotte, E., Spitzenstetter, F., Giselbrecht, D. and Muzet, A., 2004. Influence of age, speed and duration of monotonous driving task in traffic on the driver's useful visual field. Vision research, 44(23), pp.2737-2744.

[9] Käthner, I., Wriessnegger, S.C., Müller-Putz, G.R., Kübler, A. and Halder, S., 2014. Effects of mental workload and fatigue on the P300, alpha and theta band power during operation of an ERP (P300) brain-computer interface. Biological psychology, 102, pp.118-129.

[10] Bianchi-Berthouze, N., 2013. Understanding the role of body movement in player engagement. Human-Computer Interaction, 28(1), pp.40-75.

[11] Cahn, B.R. and Polich, J., 2013. Meditation states and traits: EEG, ERP, and neuroimaging studies.

[12] Wong, C.W., Olafsson, V., Tal, O. and Liu, T.T., 2013. The amplitude of the resting-state fMRI global signal is related to EEG vigilance measures. Neuroimage, 83, pp.983-990.

[13] Gola, M., Magnuski, M., Szumska, I. and Wróbel, A., 2013. EEG beta band activity is related to attention and attentional deficits in the visual performance of elderly subjects. International Journal of Psychophysiology, 89(3), pp.334-341.

[14] Laurent, F., Valderrama, M., Besserve, M., Guillard, M., Lachaux, J.P., Martinerie, J. and Florence, G., 2013. Multimoda information improves the rapid detection of mental fa- tigue. Biomedical Signal Processing and Control, 8(4), pp.400408 .

[15] Lim, J., Quevenco, F.C. and Kwok, K., 2013. EEG alpha activity is associated with individual differences in post-break improvement. Neuroimage, 76, pp.81-89.

[16] Zarjam, P., Epps, J., Chen, F. and Lovell, N.H., 2013. Estimating cognitive workload using wavelet entropy-based features during an arithmetic task. Computers in biology and medicine, 43(12), pp.2186-2195.

[17] Kortelainen, J., Jia, X., Seppänen, T. and Thakor, N., 2012. Increased electroencephalographic gamma activity reveals awakening from isofluraneanaesthesia in rats. British journal of anaesthesia, 109(5), pp.782-789.

[18] Chen, L.L., Zhao, Y., Zhang, J. and Zou, J.Z., 2015. Automatic detection of alertness/drowsiness from physiological signals using wavelet-based nonlinear features and machine learning. Expert Systems with Applications, 42(21), pp.7344-7355.

[19] Åkerstedt, T., Hallvig, D., Anund, A., Fors, C., Schwarz, J. and Kecklund, G., 2013. Having to stop driving at night because of dangerous sleepiness-awareness, physiology and behaviour. Journal of sleep research, 22(4), pp.380-388.

[20] Anderer, P., 2015. Advanced analysis of pharmaco-sleep data in humans. Neuropsychobiology, 72(3-4), pp.178-187.

[21] Lawoyin, S., 2014. Novel technologies for the detection and mitigation of drowsy driving. Virginia Commonwealth University.

[22] Nguyen, Q.N., Tho, L.T.A., Van, T.V., Yu, H. and Thang, N.D., 2017, June. Visual based drowsiness detection using facial features. In International Conference on the Development of Biomedical Engineering in Vietnam (pp. 723-727). Springer, Singapore.

[23] Mallet, Y., Coomans, D., Kautsky, J. and De Vel, O., 1997. Classification using adaptive wavelets for feature extraction. IEEE transactions on pattern analysis and machine intelligence, 19(10), pp.1058-1066.

[24] daSilveira, T.L., Kozakevicius, A.J. and Rodrigues, C.R., 2016. Automated drowsiness detection through wavelet packet analysis of a single EEG channel. Expert Systems with Applications, 55, pp.559-565.

[25] Alotaiby, T., El-Samie, F.E.A., Alshebeili, S.A. and Ahmad, I., 2015. A review of channel selection algorithms for EEG signal processing. EURASIP Journal on Advances in Signal Processing, 2015(1), p.66.

[26] Dabbu, S., Malini, M., Reddy, B.R. and Vyza, Y.S.R., 2017. ANN based Joint Time and frequency analysis of EEG for detection of driver drowsiness. Defence Life Science Journal, 2(4), pp.406-415.

[27] Youh, J., Hong, J.S., Han, D.H., Chung, U.S., Min, K.J., Lee, Y.S. and Kim, S.M., 2017. Comparison of electroencephalography (EEG) coherence between major depressive disorder (MDD) without comorbidity and MDD comorbid with Internet gaming disorder. Journal of Korean medical science, 32(7), pp.1160-1165.

[28] Piotrowski, Z. and Szypulska, M., 2017. Classification of falling asleep states using HRV analysis. Biocybernetics and Biomedical Engineering, 37(2), pp.290-301.

[29] Zhang, C. and Schmöcker, J.D., 2017. A Markovian model of user adaptation with case study of a shared bicycle scheme. Transportmetrica B: Transport Dynamics, pp.1-14.

[30] Zhang, L., Wade, J., Bian, D., Fan, J., Swanson, A., Weitlauf, A., Warren, Z. and Sarkar, N., 2017. Cognitive load measurement in a virtual reality-based driving system for autism intervention. IEEE transactions on affective computing, 8(2), pp.176-189.

[31] Sauvet, F., Bougard, C., Coroenne, M., Lely, L., Van Beers, P., Elbaz, M., Guillard, M., Leger, D. and Chennaoui, M., 2014. Inflight automatic detection of vigilance states using a single EEG channel. IEEE Transactions on Biomedical Engineering, 61(12), pp.2840-2847.

[32] Graditi, G., Ferlito, S., Adinolfi, G., Tina, G.M. and Ventura, C., 2016. Energy yield estimation of thin-film photovoltaic plants by using physical approach and artificial neural networks. Solar Energy, 130, pp.232-243.

[33] Rosipal, R., Peters, B., Kecklund, G., Åkerstedt, T., Gruber, G., Woertz, M., Anderer, P. and Dorffner, G., 2007, July. EEGbased drivers' drowsiness monitoring using a hierarchical Gaussian mixture model. In International Conference on Foundations of Augmented Cognition (pp. 294-303). Springer, Berlin, Heidelberg. 
[34] Mandal, N.K. and Pal, M., 2008. Optimum mixture design using deficiency criterion. Communications in Statistics-Theory and Methods, 37(10), pp.1565-1575.

[35] Bulling, A., Ward, J.A., Gellersen, H. and Troster, G., 2011. Eye movement analysis for activity recognition using electrooculography. IEEE transactions on pattern analysis and machine intelligence, 33(4), pp.741-753.

[36] Chang, W.D., Cha, H.S. and Im, C.H., 2016. Removing the Interdependency between Horizontal and Vertical Eye-Movement Components in Electrooculograms. Sensors, 16(2), p.227.

[37] Chen, L.L., Zhao, Y., Zhang, J. and Zou, J.Z., 2015. Automatic detection of alertness/drowsiness from physiological signals using wavelet-based nonlinear features and machine learning. Expert Systems with Applications, 42(21), pp.7344-7355.

[38] Han, W., Li, X. and Xi, J., 2018. A statistical-based approach for driving style recognition using Bayesian probability with kernel density estimation. IET Intelligent Transport Systems.

[39] Flad, N., Fomina, T., Buelthoff, H.H. and Chuang, L.L., 2015, October. Unsupervised clustering of EOG as a viable substitute for optical eye tracking. In Workshop on Eye Tracking and Visualization (pp. 151-167). Springer, Cham.

[40] Dawson, D., Searle, A.K. and Paterson, J.L., 2014. Look before you (s) leep: evaluating the use of fatigue detection technologies within a fatigue risk management system for the road transport industry. Sleep medicine reviews, 18(2), pp.141-152.

[41] Fuhl, W., Santini, T.C., Kübler, T. and Kasneci, E., 2016, March Else: Ellipse selection for robust pupil detection in real-world environments. In Proceedings of the Ninth Biennial ACM Symposium on Eye Tracking Research \& Applications (pp. 123-130). ACM.

[42] Gao, X.Y., Zhang, Y.F., Zheng, W.L. and Lu, B.L., 2015, April. Evaluating driving fatigue detection algorithms using eye tracking glasses. In Neural Engineering (NER), 2015 7th International IEEE/EMBS Conference on (pp. 767-770). IEEE.

[43] Othman, N. and Dorizzi, B., 2015. Impact of quality-based fusion techniques for video-based iris recognition at a distance. IEEE transactions on Information Forensics and Security, 10(8), pp.1590-1602.

[44] Gou, C., Wu, Y., Wang, K., Wang, K., Wang, F.Y. and Ji, Q., 2017. A joint cascaded framework for simultaneous eye detection and eye state estimation. Pattern Recognition, 67, pp.23-31.

[45] Liang, Y., Horrey, W.J., Howard, M.E., Lee, M.L., Anderson, C., Shreeve, M.S., O'Brien, C.S. and Czeisler, C.A., 2017. Prediction of drowsiness events in night shift workers during morning driving. Accident Analysis \& Prevention.

[46] McDonnell, D., Galvanic Ltd, 2017. Electrodermal Activity Sensor. U.S. Patent Application 15/124,372.

[47] McIntire, L.K., McKinley, R.A., Goodyear, C. and McIntire, J.P., 2017, May. Use of head-worn sensors to detect lapses in vigilance through the measurement of PERCLOS and cerebral blood flow velocity. In Disruptive Technologies in Sensors and Sensor Systems (Vol. 10206, p. 102060K). International Society for Optics and Photonics.

[48] Meng, S.H., Hu, S.B., Huang, A.C., Huang, T.J., Xie, Z. and Jian, C., 2017, October. Research on Eye Detection and Fatigue Early Warning Technologies. In The Euro-China Conference on Intelligent Data Analysis and Applications (pp. 3-9). Springer, Cham.

[49] M. Nagesh, Md. Zia Ur Rahman, "A New ECG Signal Enhancement Strategy using Non-Negative Algorithms", International Journal of Control Theory and Applications Vol.10, no.35, 2017, pp.323-333.

[50] G V S Karthik, Md. Zia Ur Rahman, "ECG Signal Enhancement using Circular Leaky Adaptive Algorithm in an IOT Enabled Sensor System", International Journal of Control Theory and Applications,Vol.10, no.35, 2017, pp.271-282.

[51] Md. Salman, Md. Zia Ur Rahman, "Efficient and Low Complexity Noise Cancellers for Cardiac Signal Enhancement using Proportionate Adaptive Algorithms", Indian Journal Science and Technology,Vol.9, no-37, pp. 1-11, October 2016.

[52] M. Nagesh, Md. Zia Ur Rahman, "Efficient Noise Cancellers for ECG Signal Enhancement for Telecardiology Applications", Leonardo Electronic Journal of Practices and Technologies,Issue 29, 2016, pp.79-92.

[53] M. Nagesh, Md. Zia Ur Rahman, "Efficient Cardiac Signal Enhancement Techniques Based on Variable Step Size and Data Normalized Hybrid Signed Adaptive Algorithms", International Review on Computers and Software,Vol.11, no.10, 2016, pp.872-883.
[54] B. Srikanth, Md. Zia Ur Rahman, "Efficient ECG Signal Conditioning Techniques using Variable Step Size LMF Algorithms", International Journal of Engineering and Technology,Vol. 8, No 2, pp.660-668, 2016.

[55] Asiya Sulthana, Md. Zia Ur Rahman, "Design and Implementation of Efficient Low Complexity Biomedical Artifact Canceller for Nano Devices", Leonardo Electronic Journal of Practices and Technologies,Issue 28, pp. 197-210, 2016.

[56] Md. Zia Ur Rahman, et. al., "Artifact Removal in ECG Signals using Modified Data Normalization Based Signal Enhancement Units for Health Care Monitoring Systems", Journal of Theoretical and Applied Information Technology,Vol.93, no.2, 2016, pp.540-551.

[57] Sk. Nore Johny Basha, Md Zia-Ur-Rahman, and Dr.B.V. Rama Mohana Rao, " Noise Removal from Electrocardiogram Signals using Leaky and Normalized version of Adaptive Noise Canceller," International Journal of Computer Science \& Communication Networks, ISSN: 2249-5789, Vol. 1(1), Sep.-Oct. 2011.

[58] Md. Zia Ur Rahman, S.R.Ahamed, D.V.R.K Reddy, "Efficient and Simplified Adaptive Noise Cancellers for ECG Sensor based Remote Health Monitoring", IEEE Sensors Journal, vol.12, no.3, pp. 566-573, 2012.

[59] Md. Zia Ur Rahman, S.R.Ahamed, D.V.R.K Reddy, "Efficient sign based normalized adaptive filtering techniques for cancelation of artifacts in ECG signals: Application to wireless biotelemetry", Elsevier Signal Processing, vol.91, pp. 225-239, 2011.

[60] Sowmya I, Ch. Sathi Raju, Md Zia-Ur-Rahman, D.V.R.K Reddy "Respiration Baseline wander removal from cardiac signals using an optimized Adaptive Noise canceller", Canadian Journal of Signal Processing, Vol-2, no-3, pp.27-31, 2011.

[61] Md.Zia Ur Rahman, S.R.Ahamed and D.V.R.K Reddy, "Noise Cancellation in ECG Signals using Computationally Simplified Adaptive Filtering Techniques: Application to Biotelemetry", Signal Processing: An International Journal, CSC Journals, ISSN 1985-2312, Vol. 3, Issue 5, pp. 1-12, 2009.

[62] Md. Nizamuddin Salman, P. Trinatha Rao, Md.Zia Ur Rahman, "Cardiac Signal Enhancement Using Normalised Variable Step Algorithm For Remote Healthcare Monitoring Systems," International Journal of Medical Engineering and Informatics, Inderscience Pub, Vol. 9, No. 2, 2017, pp. 145-161.

[63] Md. Zia Ur Rahman, Adaptive Noise Cancellers for Cardiac Signal Enhancement for IOT Based Health Care Systems, Journal of Theoretical and Applied Information Technology,Vol.95, no.10, 2017, pp.2206-2213. 